\title{
Genomic surveys by methylation-sensitive SNP analysis identify sequence-dependent allele-specific DNA methylation
}

\author{
Kristi Kerkel ${ }^{1}$, Alexandra Spadola ${ }^{2}$, Eric Yuan ${ }^{1}$, Jolanta Kosek ${ }^{1}$, Le Jiang ${ }^{1}$, Eldad Hod ${ }^{3}$, Kerry Li $^{1}$, \\ Vundavalli V Murty ${ }^{1,3}$, Nicole Schupf ${ }^{4}$, Eric Vilain ${ }^{5,6}$, Mitzi Morris ${ }^{7}$, Fatemeh Haghighi ${ }^{7}$ \& Benjamin Tycko ${ }^{1,3}$
}

\begin{abstract}
Allele-specific DNA methylation (ASM) is a hallmark of imprinted genes, but ASM in the larger nonimprinted fraction of the genome is less well characterized. Using methylation-sensitive SNP analysis (MSNP), we surveyed the human genome at $50 \mathrm{~K}$ and $250 \mathrm{~K}$ resolution, identifying ASM as recurrent genotype call conversions from heterozygosity to homozygosity when genomic DNAs were predigested with the methylation-sensitive restriction enzyme Hpall. Using independent assays, we confirmed ASM at 16 SNP-tagged loci distributed across various chromosomes. At 12 of these loci $(75 \%)$, the ASM tracked strongly with the sequence of adjacent SNPs. Further analysis showed allele-specific mRNA expression at two loci from this methylation-based screen-the vanin and CYP2A6-CYP2A7 gene clusters-both implicated in traits of medical importance. This recurrent phenomenon of sequence-dependent ASM has practical implications for mapping and interpreting associations of noncoding SNPs and haplotypes with human phenotypes.
\end{abstract}

Allele-specific CpG methylation (ASM) is a hallmark of genomic imprinting, but the characteristics of ASM at nonimprinted loci are less well understood. DNA methylation tracking as a genetic trait was found in early studies of human VNTR loci ${ }^{1,2}$, and a recent study using genome fractionation uncovered a $\mathrm{CpG}$ island with parent of origin-independent ASM on chromosome 21 (ref. 3). We have described a microarray-based approach, called MSNP, for genetic and epigenetic profiling ${ }^{4}$. Genomic DNA is digested with a nonmethylation-sensitive restriction enzyme with or without a second, methylation-sensitive, restriction enzyme, such as HpaII. Probes are synthesized by linker ligation and PCR and hybridized to Affymetrix SNP arrays. Fragments with internal unmethylated HpaII sites drop out from the plus-HpaII representations. Thus, by comparing the results in the minus- and plus-HpaII representations, we can measure net methylation and also detect ASM, which appears as a conversion from an $\mathrm{AB}$ genotype call to $\mathrm{BB}$ or $\mathrm{AA}$.

Here, we report genome-wide surveys for ASM using MSNP at 50K and $250 \mathrm{~K}$ resolution. The $50 \mathrm{~K}$ dataset consisted of single $\mathrm{Xba \textrm {I }}$ and $\mathrm{XbaI}+\mathrm{HpaII}$ representations from 12 normal tissue samples: peripheral blood leukocyte (PBL) DNA from four adults, kidney DNA from one adult, brain and lung DNA from one adult, DNA from three placentas (sampled at the fetal surface), and buccal cell DNA from two adults. On this 50K array, there are 39,100 class 1 SNPs (noninformative for DNA methylation, because of absence of HpaII sites within the XbaI fragment), 15,057 class 2 SNPs (informative, because of the presence of nonpolymorphic HpaII sites) and 4,328 class 3 SNPs (not reliably informative, because of polymorphic $\mathrm{HpaII}$ sites). In assessing the SNP-tagged loci for ASM, we first eliminated those with weak signals-below the 10th percentile in the XbaI representations-in $\geq 5$ samples. We next asked for allele call conversions in $\geq 3$ samples in the plus-HpaII representations, producing a list of 74 SNP-tagged loci. Lastly, we examined the hybridization quality and the selective reduction in intensity of one of the two alleles for each of these loci using the perfect match/mismatch (PM/MM) view in dChip ${ }^{5}$.

We brought forward two of the most convincing candidate loci for independent validations. SNP rs1042073, in exon 13 of the lactotransferrin $(L T F)$ gene, showed $\mathrm{AB} \rightarrow \mathrm{AA}$ conversions with HpaII predigestion in five tissues (PBL, brain, kidney, lung and placenta), each obtained from a different individual, whereas no $A B \rightarrow B B$ conversions were seen (Fig. 1a). These results predicted that the $\mathrm{C}$ allele would be consistently more methylated than the Tallele at one or both of the two HpaII sites in this XbaI fragment. As shown for several tissues from different individuals (Fig. 1), PCR/RFLP and sequencing consistently showed a reduced representation of the $\mathrm{T}$ allele when the DNA had been predigested with HpaII, and the same genotype dependence was seen in an expanded series (Table 1 and Supplementary Table 1 online). To validate this correlation between ASM and SNP genotype using a non-PCR-based method, we did DNA blotting

${ }^{1}$ Institute for Cancer Genetics, ${ }^{2}$ Department of Obstetrics and Gynecology, ${ }^{3}$ Department of Pathology and ${ }^{4}$ Sergievsky Center and Department of Epidemiology, Columbia University Medical Center, New York, New York 10032, USA. ${ }^{5}$ Department of Urology and ${ }^{6}$ Department of Human Genetics, University of California Los Angeles, Los Angeles, California 90095, USA. ${ }^{7}$ Department of Psychiatry, Columbia University Medical Center, New York, New York 10032, USA. Correspondence should be addressed to B.T. (bt12@columbia.edu). 
a

50K Xbal array: kidney Digest: SNP call:

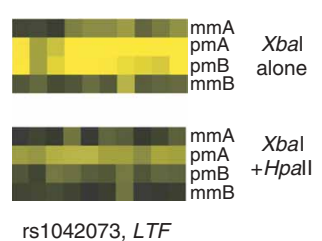

rs1042073, LTF

AA b
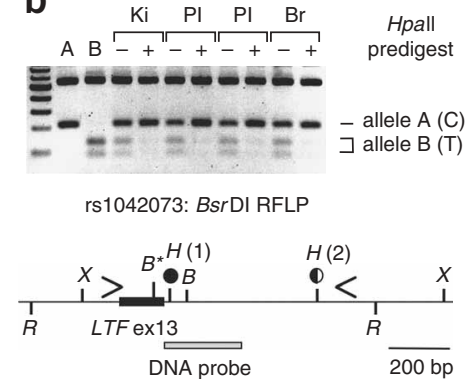

C

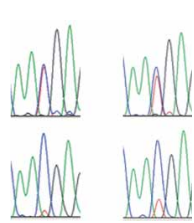

Placenta Kidney

SNP rs1042073 d

\begin{tabular}{c} 
Predigest: \\
\hline $\begin{array}{c}X b a l \\
\text { alone }\end{array}$ \\
\\
Xbal \\
+ Hpall
\end{tabular}

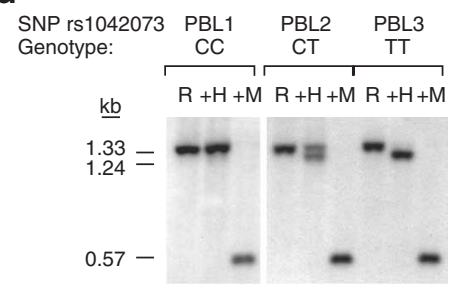

Figure 1 Genotype-dependent ASM in LTF intron 13. (a) Hybridization data from 50K Xbal MSNP. Perfect match (pm) and mismatch (mm) intensities are shown, and the genotype calls from Xbal and Xbal + Hpall genomic representations are indicated. With addition of Hpall, there is a call conversion from $A B$ to $A A$ at SNP rs1042073. (b) Predigestion followed by PCR and RFLP assay validating ASM in the Xbal fragment containing SNP rs1042073. Genomic DNA was digested with Xbal or with Xbal + Hpall, followed by PCR with the indicated primers (arrows). PCR products were digested with BsrDI to score the RFLP corresponding to SNP rs1042073 (asterisk). In each of these samples and in additional individuals (Table 1), the Tallele drops out with Hpall predigestion, indicating relative hypomethylation. The methylation status of the two Hpall sites between the primers, based on this assay and genomic DNA (Southern) blotting, is indicated by the circles (methylated, black; ASM, half-black). The image of the ethidium-stained gel is inverted to show the bands as dark on a light background. (c) Confirmation of ASM by predigestion/PCR/sequencing. The double peak indicating heterozygosity at SNP rs1042073 in the Xbal-digested samples is nearly a single peak in PCR products from the Xbal + Hpall pre-digested samples. (d) Genotype-dependent ASM downstream of LTF exon 13 indicated by DNA blotting. In a series of 21 individuals, 10 with rs 1042073 genotype CC, 8 with genotype CT and 3 with genotype TT, the ASM at Hpall site 2, seen as the doublet in the Rsal + Hpall lane, was only observed in tissues from individuals who were heterozygous: individuals homozygous for the $\mathrm{C}$ allele at this SNP showed full methylation of this Hpall site, whereas those homozygous for the Tallele lacked methylation at this site. Overall $P=1.72 \times 10^{-8}$, Fisher's exact test with null hypothesis of random allelic methylation. $B, B s r D I ; H, H p a l l ; M, M s p l ; R, R s a l ; X, X b a l$.

of genomic DNAs from 21 individuals with the 3 possible rs 1042073 genotypes. ASM was readily scored as a doublet band pattern, biallelic methylation as a single upper band, and biallelic lack of methylation as a single lower band. As shown in Figure 1d, there was an invariant relationship between the rs1042073 genotype and the pattern of methylation, with C-allele homozygotes showing biallelic methylation, CT heterozygotes showing ASM and TT homozygotes showing biallelic lack of methylation at this site. Given this notably constant relationship, we sought artifactual explanations, such as the presence of a polymorphic HpaII site created by or in linkage disequilibrium with the index SNP in the same restriction fragment. However, sequencing excluded this possibility. As ASM is often found at imprinted loci, we also sought to exclude imprinting as an explanation. We addressed this question by using trios of DNA from maternal and paternal PBL and placenta (fetal surface) from the offspring. Among the informative trios, the methylated allele in the LTF exon 13 region was paternal in origin in four but maternal in origin in one, thus excluding parental imprinting as an explanation for the ASM (Supplementary Fig. 1 online).

We obtained similar results for a second locus from the $50 \mathrm{~K}$ survey, tagged by SNP rs9258170 in the upstream region of the HLA-F gene. There were $\mathrm{AB} \rightarrow \mathrm{AA}$ call conversions with $\mathrm{HpaII}$ predigestion in each of five samples (one kidney, one lung, one brain, one placenta, and two buccal epithelial cell preparations), and no $\mathrm{AB} \rightarrow \mathrm{BB}$ conversions. As shown by pre-digestion followed by PCR, ASM in the HLA-F upstream region correlated strongly, although not invariably, with the local DNA sequence, as scored by SNPs and indels (Table 1 and Supplementary Fig. 2 online). To ask whether the ASM at the HpaII sites in this region might reflect more widespread ASM at other CpG sites, we carried out bisulfite sequencing of a region near the index SNP. This procedure showed a patch of differential methylation affecting at least $17 \mathrm{CpG}$ dinucleotides (Supplementary Fig. 2).

We next carried out MSNP using 250K StyI SNP arrays, containing 164,561 class 1 (69\%), 57,583 class 2 (24\%), and 15,906 class 3 SNPs (7\%). Our $250 \mathrm{~K}$ survey included six PBL samples, two normal bone marrow samples, one sample of $\mathrm{CD} 34^{+} \mathrm{Lin}^{-}$hematopoietic cells, and three placental samples. We analyzed duplicate technical replicates for StyI and StyI + HpaII representations, and single determinations for StyI + MspI (60 microarrays). We asked for call conversions from AB in StyI to AA or BB in StyI + HpaII in both technical replicates in at least 2 of the 12 biological samples. To exclude loci with biallelic hypomethylation, we additionally required that the average StyI + HpaII intensity be reduced not more than $70 \%$ from the average StyIonly intensity, and that the intensity in the StyI + MspI representation be at least $40 \%$ less than the mean signal with StyI + HpaII. This procedure produced a list of 58 loci as candidates for ASM. As expected, this list was depleted in class 1 SNPs $(0,0 \%)$ and enriched in class $2(31,53 \%)$ and class $3(27,47 \%)$ SNPs. The opposite analysis, asking for all class 2 SNPs with persistent $\mathrm{AB}$ calls and preserved intensity of both alleles in the plus-HpaII representations (reduced not more than $70 \%$ from the minus-HpaII intensity) in all heterozygous samples (requiring at least two of the samples to be heterozygous), with a signal intensity in the StyI + MspI representation at least $40 \%$ less than the mean signal with StyI + HpaII, yielded 15,454 class 2 SNPs. So within the sensitivity of this method and given the validations and determination of the true-positive rate below, we estimate that at least $0.16 \%$ of the informative SNP-tagged loci queried by the array have recurrent ASM in the tissues examined.

As internal controls in this $250 \mathrm{~K}$ dataset, we found consistent call conversions at SNPs in three imprinted domains: in a PBL sample at SNP rs11161318 located $0.5 \mathrm{~kb}$ downstream of the $3^{\prime}$ end of the MAGEL2 gene, in a bone marrow sample at SNP rs231907 in the KCNQ1 gene, and in the $\mathrm{CD}^{+} 4^{+}$stem cells at SNP rs2107425 upstream of the $H 19$ gene. Monoallelic expression of MAGEL2 mRNA as a result of parental imprinting has been shown previously ${ }^{6,7}$, and DNA methylation occurs preferentially on the maternal allele at multiple sites over this imprinted domain on chromosome 15 (ref. 8), but ASM has not been previously examined in the MAGEL2 downstream region. We therefore validated the MSNP findings for this SNP-tagged StyI fragment using pre-digestion followed by PCR and RFLP. As expected for an imprinted locus, in which parent-of-origin, not DNA sequence, dictates which allele 
Table 1 Sequence dependence of ASM at SNP-tagged loci from the 50K and 250K MSNP screens

\begin{tabular}{|c|c|c|c|c|c|c|c|}
\hline dbSNP ID & Gene or region & Tissue & CT $(N)$ & Allele C methyl. & Allele T methyl. & No ASM & Binomial test \\
\hline \multirow[t]{2}{*}{ rs1042073 } & LTF exon 13 & PBL & 49 & 48 & 0 & 1 & $P<10^{-8}$ \\
\hline & & Placenta & 33 & 31 & 0 & 2 & $P<10^{-8}$ \\
\hline rs6494120 & GCNT3 US & PBL & 48 & 0 & 44 & 4 & $P<10^{-8}$ \\
\hline rs12873012 & POU4F1 DS & PBL & 45 & 13 & 2 & 30 & $P=0.0032$ \\
\hline rs6969642 & DNAJB6 & PBL & 30 & 0 & 27 & 3 & $P<10^{-8}$ \\
\hline rs9366927 & FGD2- PIM1 & PBL & 9 & 9 & 0 & 0 & $P=0.002$ \\
\hline rs9951893 & PHLPP-BCL2 & PBL & 37 & 35 & 0 & 2 & $P<10^{-8}$ \\
\hline dbSNP ID & Gene & Tissue & CG $(N)$ & Allele C methyl. & Allele G methyl. & No ASM & Binomial test \\
\hline \multirow[t]{2}{*}{ rs3998799 } & $H L A-F \cup S$ & PBL & 15 & 1 & 7 & 7 & $P=0.0053 ;$ combined \\
\hline & & Placenta & 6 & 0 & 3 & 3 & \\
\hline dbSNP ID & Gene & Tissue & $\mathrm{AG}(N)$ & Allele A methyl. & Allele G methyl. & No ASM & Binomial test \\
\hline \multirow[t]{2}{*}{ rs34724660 } & CYP2 cluster $^{a}$ & Liver & 7 & 0 & 7 & 0 & $P=0.0001 ;$ combined \\
\hline & & PBL & 6 & 0 & 6 & 0 & \\
\hline rs443731 & EFNB1 DS & Placenta & 14 & 1 & 10 & 3 & $P=0.0053$ \\
\hline rs943049 & PARP4-ATP12A & PBL & 19 & 17 & 0 & 2 & $P<10^{-5}$ \\
\hline rs4925109 & $R A / 1$ intron 2 & PBL & 25 & 0 & 19 & 6 & $P<10^{-5}$ \\
\hline rs6569826 & VNN1 US & PBL & 44 & 18 & 0 & 26 & $P<10^{-5}$ \\
\hline rs206337 & N4BP2L 1 intron 1 & Placenta & 19 & 8 & 5 & 6 & n.s. \\
\hline rs11161318 & MAGEL2 DS & PBL & 9 & 4 & 5 & 0 & n.s. \\
\hline dbSNP ID & Gene & Tissue & AT $(N)$ & Allele A methyl. & Allele T methyl. & No ASM & Binomial test \\
\hline \multirow[t]{2}{*}{ rs3844442 } & CYP2 cluster $^{a}$ & PBL & 16 & 0 & 5 & 11 & $P=0.001 ;$ combined \\
\hline & & Liver & 15 & 0 & 5 & 10 & \\
\hline dbSNP ID & Gene & Tissue & indel $(N)$ & Long allele methyl. & Short allele methyl. & No ASM & Binomial test \\
\hline rs9278271 & HLA-FUS & PBL & 8 & 0 & 5 & 3 & $P=0.031$ \\
\hline \multirow[t]{2}{*}{ rs34039986 } & CYP2A7 exon $2 b$ & PBL & 11 & 10 & 0 & 1 & $P=0.000031 ;$ combined \\
\hline & & Liver & 9 & 5 & 0 & 4 & \\
\hline
\end{tabular}

IDs refer to the SNP used for scoring ASM by Hpall predigestion followed by PCR, sizing gel analysis and/or direct sequencing. Reported are exact $P$ values from two-tail binomial tests testing the null hypothesis of random allelic methylation. Samples with no ASM were not included in the statistical tests. Index SNPs that create or destroy CpG sites are LTF rs1042073, VNN1 US rs6569826 and CG018 intron 1 rs206337; the index SNPs do not affect Hpall sites (the enzyme used to score ASM). Methyl., relative hypermethylation of one allele; combined, pooled data from both tissues; $N$, number of heterozygotes; n.s., not significant; US, upstream; DS, downstream. See Supplementary Table 1 for additional features of ASM at 16 validated loci.

${ }^{a}$ ASM in this region of the CYP2Ab-CYP2A7 cluster was haplotype dependent in human livers: samples from individuals heterozygous for SNP rs34724660 all showed ASM, whereas those that were heterozygous for the nearby SNP rs3844442 but homozygous for rs34724660 did not show ASM.

becomes methylated, ASM in the MAGEL2 region did not correlate with the SNP genotype (Supplementary Fig. 3 online).

We next selected a group of loci reported as having ASM in the $250 \mathrm{~K}$ data, outside of known imprinted regions, for independent validations in larger series of individuals. These assays validated ASM for 14/18 candidate loci examined (true-positive rate 78\%). For the minority of loci that could not be validated, it was evident from PCR that biallelic hypomethylation, with relatively greater sensitivity of the SNP array probe sets for one allele, accounted for the false-positive call conversions. The MSNP data and validations by pre-digestion followed by PCR and bisulfite sequencing are shown for the CYP2A7 and VNN1 genes in Figures 2 and 3, and examples of validations of ASM at additional SNP-tagged loci are shown in Supplementary Figures 3-5 online. In the combined $50 \mathrm{~K}$ and $250 \mathrm{~K}$ data, the ASM correlated strongly with the allele in cis at the index SNP or closely adjacent informative SNPs for 12/16 (75\%) of the validated loci (Table 1 and Supplementary Table 1).

The known relationship between DNA methylation and gene expression raised the question of whether this sequence-dependent ASM might be associated with allele-specific mRNA expression (ASE).
Among four loci that we tested by comparing allelic representation in cDNA versus genomic DNA, only rare individuals showed ASE of the $L T F$ and HLA-F genes, whereas many showed strong ASE of the CYP2A7 and VNN1 genes. Both of these positive examples are in gene clusters, on chromosome 19 and 6, respectively, which lack classical upstream CpG islands and show tissue specific expression. Although our MSNP screen was done using hematopoietic tissues, we directly assayed ASM and ASE for CYP2A7 in the main expressing organ, the liver. Comparison of allelic representation in genomic and CDNA PCR products from the CYP2A7 gene showed strong ASE in 10 of 17 heterozygous human liver samples examined, with relative overexpression of the same allele in each of these 10 individuals, and sequencing across multiple intragenic SNPs revealed that the presence or absence of ASE was strongly dependent on genotype (Fig. 2b and Supplementary Table 2 online). For VNN1, we confirmed ASM by predigestion followed by RFLP analysis near the index SNP and by bisulfite sequencing across a CG-rich region, not dense enough to qualify as a CpG island, between the index SNP and the first exon (Fig. 3a,c,d). We found a consistent difference in methylation patterns between the two alleles, already detectable early in the 
a

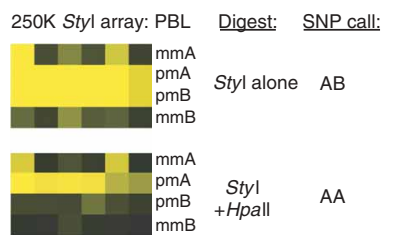

rs3844442, CYP2G1P-CYP2B7P1 b

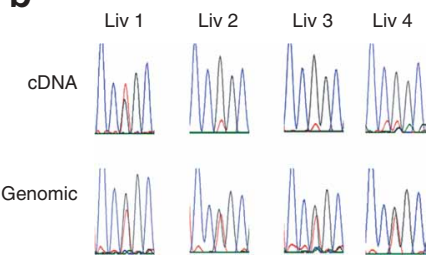

CYP2A7 exon 2b: rs3815710

Figure 2 Genotype-dependent ASM and ASE in the CYP2 gene cluster. (a) Hybridization data from 250K MSNP showing ASM within the Styl fragment containing SNP rs3844442 in PBL. See Table 1 for sequence dependence of the ASM at this location in the CYP2 gene cluster, queried by SNPs rs3844442 and rs34724660. (b) ASE of CYP2A7 mRNA in human livers. Liver DNAs were screened for heterozygosity at SNP rs3815710, and 17 heterozygous samples from different individuals were analyzed for ASE: 10 showed strongly preferential expression of the C allele (Liv2, Liv3 and Liv4, for example) and 7 showed equal biallelic expression or a slight preferential expression of the A allele (Liv1). As shown in Supplementary Table 2, the presence or absence of ASE was significantly associated with the genotype at SNP rs3815706 in CYP2A7 intron 3. Sequencing of cloned partial cDNAs showed that the two common haplotypes in this highly polymorphic gene differ by $\geq 6$ amino acids in the region spanning exon 2 a to exon 6 , suggesting that the allelic expression bias may have functional consequences (Supplementary Table 2 and data not shown). (c) Confirmation of ASM in the Styl fragment containing the index SNP in human livers by predigestion/PCR/sequencing. The double peak indicating heterozygosity at SNP rs34724660 becomes nearly a single peak in PCR products from the Hpall predigested samples. (d) ASM shown by bisulfite sequencing at two positions (gray rectangles) in the CYP2 gene cluster. The bisulfite clones were assigned to each allele in the index region using SNP rs34724660; the clones from the intragenic CpG island overlapping exon $2 \mathrm{~b}$ of the CYP2A7 gene were assigned using SNP rs3815710. Dashes in the bisulfite sequences indicate polymorphic CpG sites present on one allele and absent on the other.

hematopoietic lineage $\left(\mathrm{CD} 34^{+} \mathrm{Lin}^{-}\right.$cells; Fig. 3d). By comparing cDNA and genomic PCR products in PBL, we documented a domain-wide effect, with ASE strongest for VNN1 but also evident for the other two genes (VNN3 and VNN2) in this cluster (Fig. 3b). Both of these examples are medically relevant: prior genotypephenotype correlations for CYP2A6 (located immediately downstream of CYP2A7 and 93\% identical in amino acid sequence) have shown that genes in this cytochrome P450 cluster control blood nicotine levels and may thereby influence smoking behavior ${ }^{9}$, and a recent report described total VNN1 mRNA expression as a quantitative trait linked to haplotypes, and in turn to HDL cholesterol levels ${ }^{10}$. Future methylation-based screens using the methods developed here will likely uncover additional examples of sequence-dependent ASM linked to important human traits.

This dependence of ASM on the local DNA sequence in cis is distinct from other epigenetic phenomena such as imprinting, allelic exclusion, random monoallelic methylation and expression ${ }^{11-13}$, and epimutation (which may be haplotype-dependent in some cases ${ }^{14-17}$ ). Mechanisms leading to sequence-dependent ASM will likely differ among loci: allele-specific affinity for DNA-binding proteins with downstream effects on DNA methylation is one obvious possibility and our findings, and in conjunction with prior data ${ }^{10,18-20}$, suggests that this model may apply to the CYP2 and vanin gene clusters. Direct effects of the DNA sequence on propensity for methylation are another a

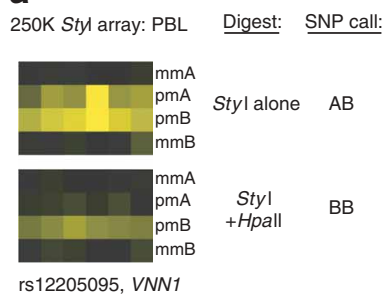

b

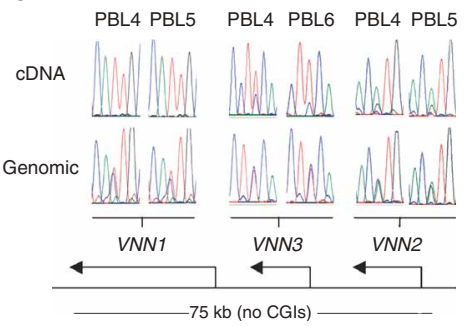

c

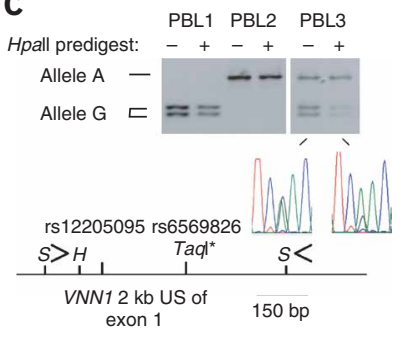

d
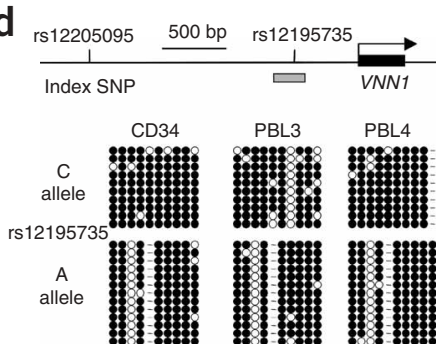

Figure 3 Genotype-dependent ASM in the VNN1 gene and allele-specific mRNA expression of all three genes in the vanin cluster. (a) Hybridization data from 250K MSNP showing ASM in the Styl fragment containing the index SNP rs12205095 in PBL. (b) ASE of three genes in the vanin cluster. Of 47 PBL samples screened for the rs2294757 SNP in VNN1 exon 1, 24 were heterozygous. Of these, 17 were analyzed for ASE: 16 showed strongly preferential expression of the A allele (PBL4 and PBL5, for example; sequence chromatograms are in the reverse direction), whereas only one sample showed equal biallelic expression. In a smaller series of heterozygotes, ASE was also observed for VNN3 and VNN2: of seven heterozygous samples examined for ASE of VNN2, four showed preferential expression of the A allele (SNP rs1883613), whereas one showed preferential expression of the G allele and two showed equal biallelic expression; of six heterozygous samples examined for ASE of VNN3, all showed preferential expression of the A allele (SNP rs2294759; sequence chromatograms are in the reverse direction). (c) Predigestion followed by PCR and RFLP and sequencing validating ASM at a Hpall site in the Styl fragment containing SNP rs12205095. Genomic DNA was mock-digested or digested with Hpall, followed by PCR with the indicated primers (arrows). PCR products were digested with Taql to score the RFLP corresponding to SNP rs6569826 (asterisk). In PBL3 and in additional individuals (Table 1), the G allele drops out with Hpall predigestion, indicating relative hypomethylation. PBL1 and PBL2 are from homozygotes. (d) ASM in the VNN1 promoter region shown by bisulfite sequencing. The alleles were distinguished by scoring SNP rs12195735 in each clone. The CD34+Lin ${ }^{-}$DNA sample and PBL3 and PBL4 are from heterozygotes with the $\mathrm{AC}$ genotype. In this region, located $0.5 \mathrm{~kb}$ from exon 1 , all three samples show a specific pattern of $\mathrm{CpG}$ methylation on the A allele, with a different pattern on the $\mathrm{C}$ allele. Dashes indicate polymorphic $\mathrm{CpG}$ sites present on one allele and absent on the other. 
possibility ${ }^{21}$. In practical terms, the phenomenon of sequence-dependent ASM extends the concept of haplotypes to include epigenetic marks ('epihaplotypes') and, as an indicator for the presence of nearby regulatory polymorphisms that confer the allelic asymmetry, it will likely be useful for fine mapping and interpreting associations of noncoding SNPs with complex genetic diseases.

\section{METHODS}

MSNP procedure. Genomic DNA from normal human tissues, obtained with institutional review board approval and anonymous to individual identifiers, was purified using the DNeasy Blood and Tissue Kit (Qiagen). For both $50 \mathrm{~K}$ $\mathrm{XbaI}$ and 250K StyI MSNP, we followed the recommended protocol of the SNP array manufacturer (Affymetrix), but with the addition of an initial HpaII or MspI digestion for the plus-HpaII and plus-MspI probes. Genomic DNA (250 ng per representation) was digested with $H p a I I$ or $M s p I$ in a total volume of $12 \mu \mathrm{l}$ (Buffer 1; New England Biolabs) for $3 \mathrm{~h}$, followed by adjustment of the buffer by addition of $15.8 \mu \mathrm{l}$ of water and $3.2 \mu \mathrm{l}$ of $10 \times$ Buffer 3 (New England Biolabs), and a further digestion with StyI for $3 \mathrm{~h}$. StyI linkers were ligated to the restriction fragments, and PCR was carried out for 30 cycles with linker primers. We carried out PCR purification using the DNA Amplification CleanUp Kit (Clontech), and probe fragmentation (brief exposure to DNase), biotinlabeling and hybridization as described in the Affymetrix protocol. We determined intensity values by normalization and model-based expression (PM-only method) using dChip ${ }^{5}$. The main determinant of the data quality is the starting material: the genomic DNA must be high molecular weight. Call rates were in the range of $89-95 \%$ with StyI and StyI + HpaII, with lower call rates of $76-88 \%$, as predicted, in the StyI $+M s p \mathrm{I}$ representations, in which many of the class 2 SNPs dropped out as a result of complete digestion of internal CCGG sites.

Independent assays for ASM. For the HpaII predigestion followed by PCR, RFLP and sequencing assays, genomic DNA, $1 \mu \mathrm{g}$ in $80 \mu \mathrm{l}$, was digested with $5 \mathrm{U}$ of HpaII overnight, followed by an additional $5 \mathrm{U}$ of HpaII for $1 \mathrm{~h}$ or incubated in parallel with buffer alone. Of this digested DNA, $25 \mathrm{ng}$ was used for each PCR (primers in Supplementary Table 3 online). In this predigestion/ PCR assay, we did a control with MspI predigestion, and verified that this procedure markedly reduce the amount of PCR product obtained under the same cycling conditions. For bisulfite conversion followed by PCR, cloning and sequencing, we treated genomic DNA with sodium bisulfite and purified the converted DNA using the CpGenome DNA modification kit (Chemicon) according to the protocol of the manufacturer. The bisulfite/PCR primers and SNP ID numbers for distinguishing the alleles in the HLA-F upstream region, two positions in the CYP2 gene cluster, and in the promoter region of the VNN1 gene are listed in Supplementary Table 3. Bisulfite PCR products were cloned in the pCR 2.1-TOPO vector (Invitrogen), and multiple clones were sequenced. For methylation-sensitive DNA (Southern) blotting of the LTF exon 13 region, we digested $3 \mu \mathrm{g}$ genomic DNA with the indicated restriction enzymes overnight, followed by electrophoresis on $1 \%$ agarose gels, transfer to Nytran membranes (Schleicher and Schull) and hybridization of the blots with a ${ }^{32}$-labeled LTF probe. The probe DNA was synthesized by genomic PCR; the primer sequences are in Supplementary Table 3.

Assays for allele-specific mRNA expression. Reverse transcription PCR was carried out with cDNAs prepared from total PBL RNA (Protoscript First Strand cDNA Synthesis Kit, New England Biolabs), using primers specific for VNN1, $V N N 2, V N N 3$ and CYP2A7, designed to span at least one intron and include at least one exonic SNP with a high minor allele frequency. We avoided SNPs within the primer sequences and sequences that were identical among family members, conditions which put strong constraints on primer placement in these highly polymorphic gene clusters. The controls for these analyses were genomic PCR products spanning these same SNPs. The primer sequences are listed in Supplementary Table 3.
Accession codes. NCBI GEO: data have been deposited with accession code GSE11409.

Note: Supplementary information is available on the Nature Genetics website.

\section{ACKNOWLEDGMENTS}

This work was supported by grants to B.T. from the US National Institutes of Health, the March of Dimes and the Leukemia and Lymphoma Society in collaboration with the Douglas Kroll Research Program.

\section{AUTHOR CONTRIBUTIONS}

Study design: K.K. and B.T. MSNP, molecular validations and analysis of ASE: K.K., A.S., E.Y., J.K., L.J., E.H., K.L. and V.V.M. Bioinformatic analyses: E.H., M.M. and F.H. Statistical analyses: N.S. and F.H. Provision of biological samples: A.S., V.V.M., N.S., E.V. and B.T. Interpretation and writing the paper: K.K., N.S., E.V. and B.T.

Published online at http://www.nature.com/naturegenetics/

Reprints and permissions information is available online at http://npg.nature.com/ reprintsandpermissions/

1. Chandler, L.A., Ghazi, H., Jones, P.A., Boukamp, P. \& Fusenig, N.E. Allele-specific methylation of the human c-Ha-ras-1 gene. Cell 50, 711-717 (1987).

2. Silva, A.J. \& White, R. Inheritance of allelic blueprints for methylation patterns. Cell 54, 145-152 (1988).

3. Yamada, Y. et al. A comprehensive analysis of allelic methylation status of $\mathrm{CpG}$ islands on human chromosome 21q. Genome Res. 14, 247-266 (2004).

4. Yuan, E. et al. A single nucleotide polymorphism chip-based method for combined genetic and epigenetic profiling: validation in decitabine therapy and tumor/normal comparisons. Cancer Res. 66, 3443-3451 (2006).

5. Lin, M. et al. dChipSNP: significance curve and clustering of SNP-array-based loss-ofheterozygosity data. Bioinformatics 20, 1233-1240 (2004).

6. Boccaccio, I. et al. The human MAGEL2 gene and its mouse homologue are paternally expressed and mapped to the Prader-Willi region. Hum. Mol. Genet. 8, 2497-2505 (1999).

7. Lee, S. et al. Expression and imprinting of MAGEL2 suggest a role in Prader-willi syndrome and the homologous murine imprinting phenotype. Hum. Mol. Genet. 9, 1813-1819 (2000).

8. Saitoh, S. et al. Clinical spectrum and molecular diagnosis of Angelman and PraderWilli syndrome patients with an imprinting mutation. Am. J. Med. Genet. 68, 195-206 (1997).

9. Tyndale, R.F. Genetics of alcohol and tobacco use in humans. Ann. Med. 35, 94-121 (2003).

10. Goring, H.H. et al. Discovery of expression QTLs using large-scale transcriptional profiling in human lymphocytes. Nat. Genet. 39, 1208-1216 (2007).

11. Gimelbrant, A., Hutchinson, J.N., Thompson, B.R. \& Chess, A. Widespread monoallelic expression on human autosomes. Science 318, 1136-1140 (2007).

12. Ohlsson, R., Tycko, B. \& Sapienza, C. Monoallelic expression: 'there can only be one'. Trends Genet. 14, 435-438 (1998).

13. Wang, J., Valo, Z., Smith, D. \& Singer-Sam, J. Monoallelic expression of multiple genes in the CNS. PLOS ONE 2, e1293 (2007).

14. Chan, T.L. et al. Heritable germline epimutation of $\mathrm{MSH} 2$ in a family with hereditary nonpolyposis colorectal cancer. Nat. Genet. 38, 1178-1183 (2006).

15. Zogel, C. et al. Identification of cis- and trans-acting factors possibly modifying the risk of epimutations on chromosome 15. Eur. J. Hum. Genet. 14, 752-758 (2006).

16. Suter, C.M. \& Martin, D.I. Inherited epimutation or a haplotypic basis for the propensity to silence? Nat. Genet. 39, 573; author reply 576 (2007).

17. Murrell, A. et al. An association between variants in the IGF2 gene and BeckwithWiedemann syndrome: interaction between genotype and epigenotype. Hum. Mol. Genet. 13, 247-255 (2004).

18. von Richter, O. et al. Polymorphic NF-Y dependent regulation of human nicotine C-oxidase (CYP2A6). Pharmacogenetics 14, 369-379 (2004).

19. Pitarque, M. et al. Identification of a single nucleotide polymorphism in the TATA box of the CYP2A6 gene: impairment of its promoter activity. Biochem. Biophys. Res. Commun. 284, 455-460 (2001).

20. Pitarque, M. et al. A nicotine C-oxidase gene (CYP2A6) polymorphism important for promoter activity. Hum. Mutat. 23, 258-266 (2004).

21. Cokus, S.J. et al. Shotgun bisulphite sequencing of the Arabidopsis genome reveals DNA methylation patterning. Nature 452, 215-219 (2008). 